\title{
Visual Experience Is Necessary for the Development of Multisensory Integration
}

\author{
Mark T. Wallace, Thomas J. Perrault Jr, W. David Hairston, and Barry E. Stein \\ Department of Neurobiology and Anatomy, Wake Forest University School of Medicine, Winston-Salem, North Carolina 27157
}

\begin{abstract}
Multisensory neurons and their ability to integrate multisensory cues develop gradually in the midbrain [i.e., superior colliculus (SC)]. To examine the possibility that early sensory experiences might play a critical role in these maturational processes, animals were raised in the absence of visual cues. As adults, the SC of these animals were found to contain many multisensory neurons, the large majority of which were visually responsive. Although these neurons responded robustly to each of their cross-modal inputs when presented individually, they were incapable of synthesizing this information. These observations suggest that visual experiences are critical for the SC to develop the ability to integrate multisensory information and lead to the prediction that, in the absence of such experience, animals will be compromised in their sensitivity to cross-modal events.
\end{abstract}

Key words: cross-modal; dark rearing; multimodal; plasticity; subcortical; superior colliculus

\section{Introduction}

Neurons in the superior colliculus (SC) have the remarkable capacity to integrate information from the different senses, a process that substantially enhances the salience of the initiating event (Meredith and Stein 1983, 1985, 1986; King and Palmer, 1985; Wallace et al., 1996, 1998; Frens and Van Opstal 1998; Bell et al., 2001; Perrault et al., 2003). Such multisensory integration appears to be of great utility in effecting orientation behavior to minimally effective environmental cues (Stein et al., 1988, 1989). However, multisensory integration is not an innate capability of SC neurons. Multisensory neurons are absent in the SC of the newborn cat (Stein et al., 1973; Wallace and Stein, 1997), and, when such neurons do appear several weeks after birth, they lack the ability to integrate their inputs from different sensory modalities (Wallace and Stein, 1997, 2000). It is only after several additional weeks of postnatal life that neurons begin to acquire this capacity, and it is not until several months later that the normal complement of multisensory integrating neurons is achieved (Wallace et al., 1997). These developmental processes appear to be gated by the maturation of influences from the cerebral cortex (Wallace and Stein, 2000, Jiang et al., 2001).

The period of early postnatal maturation preceding the onset of multisensory integration is one of rapid axonal elaboration, dendritic remodeling, and synapse formation (Sanes et al., 2000). It is also the period in which sensory experience has its greatest effect on neuronal architecture. Many studies have documented the profound impact of the experiences gathered during this maturational period on a wide variety of sensory capabilities (Kandel

Received June 25, 2004; revised Sept. 18, 2004; accepted Sept. 21, 2004

This work was funded by National Institutes of Health Grants NS36916 and MH63861. We thank Nancy Stein for editorial assistance.

Correspondence should be addressed to Mark T. Wallace, Department of Neurobiology and Anatomy, Wake Forest University School of Medicine, Winston-Salem, NC 27157. E-mail: mwallace@wfubmc.edu.

DOI:10.1523/JNEUROSCI.2535-04.2004

Copyright $\odot 2004$ Society for Neuroscience $\quad$ 0270-6474/04/249580-05\$15.00/0 et al., 2000). What is not evident from these studies is whether these experiences, so critical for the normal development of individual sensory systems, as well as for the normal cross-modal topography seen in multisensory structures (King et al., 1988; Knudsen and Brainard, 1991; Benedetti and Ferro, 1995), play any role in the development of the ability of the brain to synthesize information from different senses. The present study examined this issue by testing whether the absence of early visual experience would have any effect on the development of multisensory neurons in the SC and/or on their ability to integrate multisensory information.

\section{Materials and Methods}

Experimental groups. Cats $(n=9)$ were deprived of all visual experiences by rearing them in a light-tight environment from birth. In all other respects, the housing environment of the animals was the same as for the animals used as the control group (see below). Daily care, observations, and routine veterinary procedures were all conducted using binocular infrared goggles. In addition, an infrared viewing system allowed the animals to be monitored from an adjacent room. All physiological recordings were conducted while the animals were still housed in the dark, and all efforts were made to preclude any confounding visual experiences by anesthetizing animals for recording in their housing cages (i.e., in the dark), maintaining anesthesia throughout experimentation, and fitting the animals with occluding lenses and masks during transport and recovery. All dark-reared data were compared with those generated from agematched animals $(n=3)$ reared in a "normally" illuminated housing environment (Wallace and Stein, 1997).

Procedures. In four of the animals, recording experiments were not initiated until the animals had reached adulthood. In these animals, a series of recording experiments were conducted in a semichronic manner after the implantation of a recording chamber over the SC. In the five other animals, acute recording experiments were done at 2, 4, 6, 8, and 12 weeks after birth. All procedures were performed in compliance with the Guide for the Care and Use of Laboratory Animals (National Institutes of Health publication number 91-3207) at The Wake Forest University School of Medicine, which is accredited by the American Association for 
Accreditation of Laboratory Animal Care. Details of surgery, recording procedures, and data analyses are similar to those used previously in both developing and adult animals (Meredith and Stein, 1986; Wallace et al., 1993; Wallace and Stein, 1997) and will be described only briefly here.

Implantation and recording procedures. Animals were rendered tractable with ketamine hydrochloride (5-25 mg/kg, i.m.) and acepromazine maleate $(0.2-0.4 \mathrm{mg} / \mathrm{kg}$, i.m.). To minimize light exposure during transport to the experimental room, animals were fitted with opaque contact lenses and were also masked using heavy black felt cloth. Surgical anesthesia was maintained with halothane $(1.0-4.0 \%)$, and each animal was intubated and artificially ventilated. Body temperature and expiratory $\mathrm{CO}_{2}$ were monitored and maintained within normal physiological bounds. A craniotomy was made to allow access to the SC, and a head holder was attached to the cranium using stainless steel screws and orthopedic cement to hold the animal during recording without obstructing the face and ears. Postoperative care (i.e., analgesia and antibiotic treatment) was done in close consultation with veterinary staff.

For recording, animals were anesthetized (induction with ketamine, $5-25 \mathrm{mg} / \mathrm{kg}$, i.m., and acepromazine, $0.2-0.4 \mathrm{mg} / \mathrm{kg}$, i.m., followed by maintenance with ketamine, $5-10 \mathrm{mg} \cdot \mathrm{kg}^{-1} \cdot \mathrm{hr}^{-1}$, i.v. and acepromazine, $0.05-0.1 \mathrm{mg} \cdot \mathrm{kg}^{-1} \cdot \mathrm{hr}^{-1}$, i.v.), artificially respired, paralyzed (pancuronium bromide, $0.2 \mathrm{mg} \cdot \mathrm{kg}^{-1} \cdot \mathrm{hr}^{-1}$, i.v.) to prevent ocular drift, and administered fluids (lactated Ringer's solution, $4-8 \mathrm{cc} / \mathrm{hr}$, i.v.). Parylene-insulated tungsten electrodes $(Z=1-3 \mathrm{M} \Omega)$ were advanced through the SC using an electronically controlled hydraulic microdrive. Single-unit neural activity was recorded and amplified and was routed to an oscilloscope, audio monitor, and computer for off-line analyses.

Search stimuli, receptive field mapping, and assessment of overlap. Each neuron was tested for responses to a battery of visual, auditory, and somatosensory search stimuli. If a neuron was responsive to stimuli within a given modality, its receptive field was mapped using conventional methods (Meredith and Stein, 1986; Wallace et al., 1993). Receptive fields were transferred to standardized representations of visual, auditory, and somatosensory space (Stein and Meredith, 1993). After the initial modality classification, assessment of response specificity, and determination of receptive fields, each neuron was subjected to quantitative sensory tests (see below).

Once transformed onto standardized representations, visual and auditory receptive fields sizes were calculated by multiplying the azimuth and elevation components to arrive at an areal approximation. For somatosensory receptive fields, receptive fields sizes were determined by planimetrically measuring the area delimited on the body map representation. This representation was scaled to approximate the surface area of a standard size cat. For comparison of receptive field sizes within a modality (e.g., visual receptive fields in normal vs dark-reared animals), neurons at similar locations in the SC were evaluated. In each multisensory neuron, a calculation was made to determine the amount of overlap between the respective receptive fields. In this procedure, the mapping templates for visual, auditory, and somatosensory space were transformed into an integrated multisensory representation (Stein and Meredith, 1993). Receptive field overlap was defined as the area of commonality between the receptive fields for both modalities, expressed as a proportionate measure of the area of the largest receptive field.

Quantitative multisensory tests. A multisensory neuron was defined as one that responded to cues from more than one sensory modality or whose responses to one modality were significantly altered by the presence of a stimulus from another modality. Once the modality selectivity of a multisensory neuron (e.g., visual-auditory) was established, its responses to each single-modality stimulus and the multisensory combination were evaluated quantitatively using reproducible, electronically controlled stimuli (Meredith and Stein, 1986; Wallace et al., 1993; Wallace and Stein 1997). Each modality-specific stimulus and stimulus combination was presented $8-20$ times at $8-12 \mathrm{sec}$ interstimulus intervals, with the different conditions randomly interleaved. A multisensory interaction was operationally defined as a significant (two-tailed $t$ test; $p<$ $0.05)$ increase or decrease in the number of impulses to the combined stimuli when compared with the response to the most effective singlemodality stimulus. The magnitude of this multisensory response enhancement or depression was calculated as $\left[\left(\mathrm{CM}-\mathrm{SM}_{\max }\right) /\left(\mathrm{SM}_{\max }\right)\right] \times$
$100=\%$ interaction, in which $\mathrm{CM}$ is the mean response evoked by the combined-modality stimulus, and $\mathrm{SM}_{\max }$ is the mean response evoked by the most effective single-modality stimulus.

Histology. For each electrode penetration, the depth of every recorded neuron was noted, and electrolytic lesions (5-10 $\mu \mathrm{A}$ DC current; $10 \mathrm{sec}$ ) were made at several locations. After the final experiment, the animal was killed (sodium pentobarbital, $100 \mathrm{mg} / \mathrm{kg}$, i.v.) and perfused transcardially with saline, followed by Formalin. The midbrain was blocked stereotaxically, removed, and placed in sucrose overnight. Frozen coronal sections (50 $\mu \mathrm{m}$ thickness) were taken and counterstained with neutral red to facilitate the reconstruction of the tissue and electrode penetrations.

\section{Results}

When examined as adults, dark rearing was found to have no effect on the incidence of sensory-responsive neurons in the multisensory (i.e., below stratum opticum) layers of the SC. The incidence of sensory-responsive neurons was 2.78 per mm of electrode traverse in dark-reared animals compared with 3.02 in animals reared under normal lighting conditions ( $t$ test; $p>$ $0.05)$. Despite a sharply lower incidence of modality-specific visual neurons and a corresponding increase in the incidence of modality-specific auditory and somatosensory neurons, the overall changes in the multisensory representation brought about by dark rearing were modest (Fig. 1). As in animals reared under

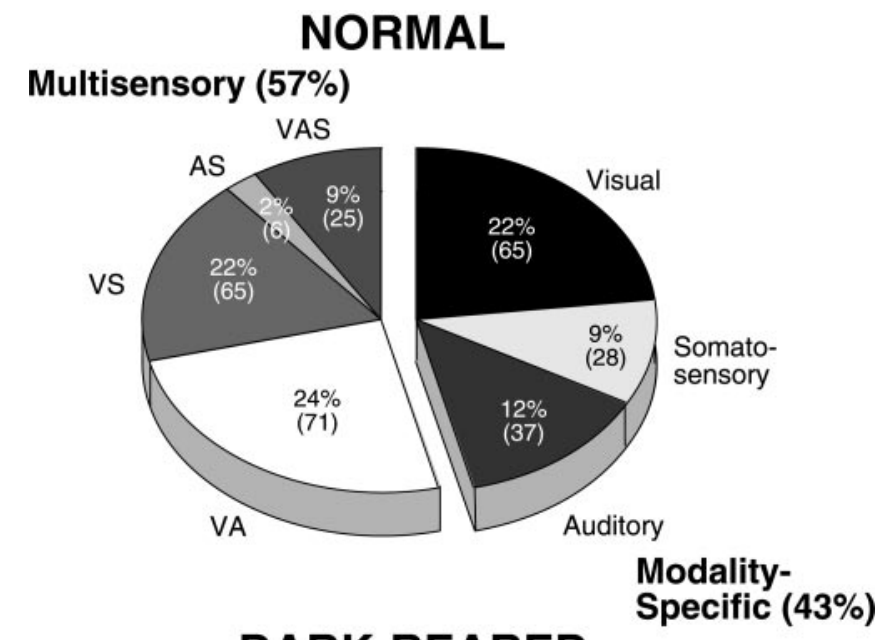

DARK-REARED

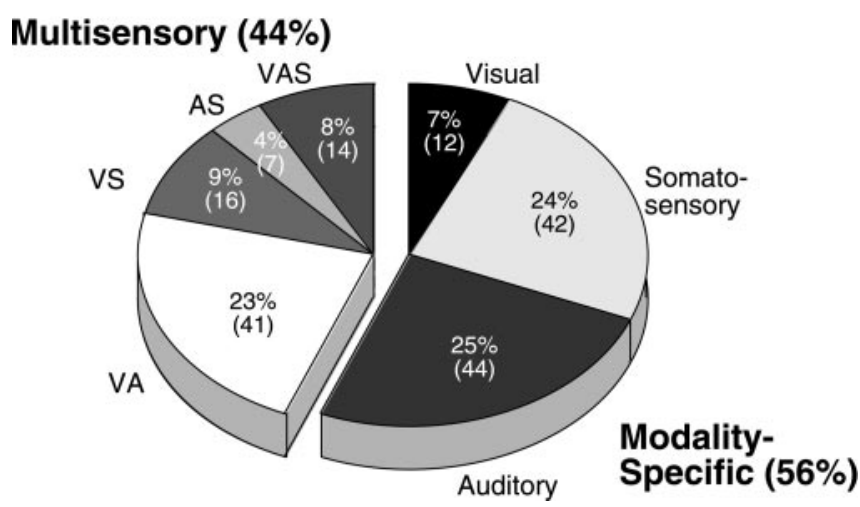

Figure 1. Dark rearing alters the distribution of sensory-responsive neurons in the SC. The top pie chart shows the distribution of modality-specific and multisensory neurons in the adult SC of animals raised under conditions of normal illumination. The bottom pie chart shows these distributions in animals raised in the absence of visual experience. Numbers in parentheses represent the total number of neurons in each category. VA, Visual-auditory; VS, visual-somatosensory; AS, auditory-somatosensory; VAS, visual-auditory-somatosensory. 
normal lighting conditions, visually responsive multisensory neurons comprised $>90 \%$ of the total multisensory population. In both groups, multisensory neurons were most heavily concentrated in the intermediate gray layers of the SC.

As opposed to its comparatively modest effect on the incidence and convergence patterns of multisensory SC neurons, dark rearing had a substantial effect on the developmental contraction in the size of their receptive fields (as well as on the receptive fields of modality-specific neurons) (Fig. 2). The large size of the receptive fields in dark-reared animals was not restricted to the visual system but was seen in auditory and somatosensory receptive fields as well (Fig. 2). Although recorded in the adult, the sizes of these receptive fields closely resembled those seen in the SC of 2- to 4-week-old animals (Wallace and Stein, 1997). Nonetheless, despite their increased size, the receptive fields in dark-reared animals were topographically organized, albeit with substantially lower spatial fidelity than that observed in control animals.

The most striking difference between SC neurons in dark-reared and control animals was seen in their responses to multisensory stimuli. Whereas multisensory SC neurons in control animals typically respond with substantial enhancements of activity when presented with spatially and temporally congruent crossmodal stimuli (Fig. 3A), this was rarely encountered ( 7 of 117) in multisensory neurons of adults who had been raised in the dark (Fig. 3B,C). Rather, the responses of these neurons to multisensory stimulus combinations were typically no different from their responses to one of the modalityspecific constituent stimuli, reflecting a lack of multisensory integration. Surprisingly, this failure was not specific to visually responsive multisensory neurons but was seen in nonvisually responsive (i.e., auditory-somatosensory) neurons as well (Fig. 3C), suggesting a general failure of SC neurons to develop the ability to integrate cross-modal information. To put these findings in context, it should be pointed out that the probability that an individual multisensory SC neuron will exhibit response enhancement in control adult animals is 0.81 . If dark rearing had no effect on the development of multisensory integration, the expectation would be that 87 (rather than 7) neurons would have shown this capacity.

One possible explanation for the paucity of demonstrable multisensory integration in these neurons is that dark rearing in some way alters the physical

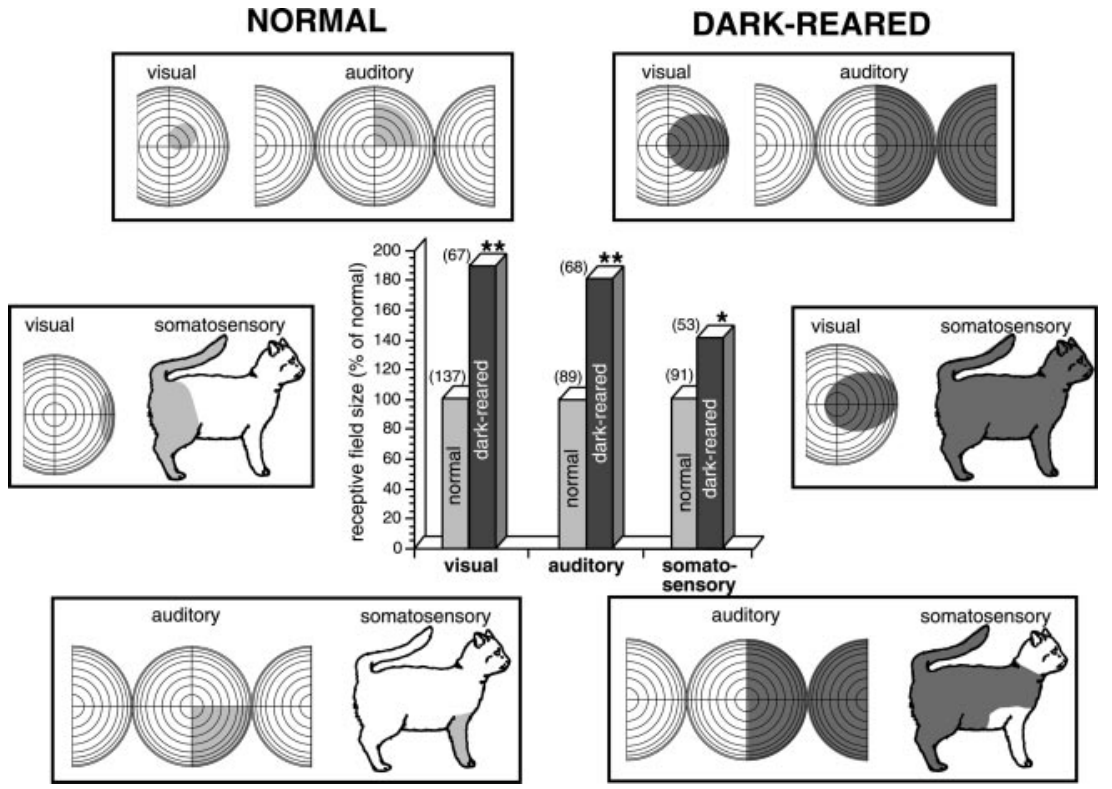

Figure 2. Receptive fields in dark-reared animals are significantly larger than those in control animals. Receptive fields (shading) are shown for three representative multisensory neurons in control (left) and dark-reared (right) animals. In the conventions shown, visual space and visual receptive fields are shown on a truncated hemisphere in which each concentric circle represents $10^{\circ}$ of visual angle. The intersection of the horizontal and vertical meridians is the point directly in front of the animal. For auditory space, the central hemisphere represents frontal auditory space, and the two half-hemispheres represent caudal space (for purposes of 2-dimensional rendering, they have been split and folded forward). The central bar graph plots the relative sizes for the visual, auditory, and somatosensory receptive fields of multisensory neurons in the SC of normal (gray bars) and dark-reared (black bars) animals. Note that these mean measures are standardized to receptive field size in normal adults (100\%). Values in parentheses represent the number of neurons in each group. ${ }^{*} p<0.05 ;{ }^{*} p<0.01$.
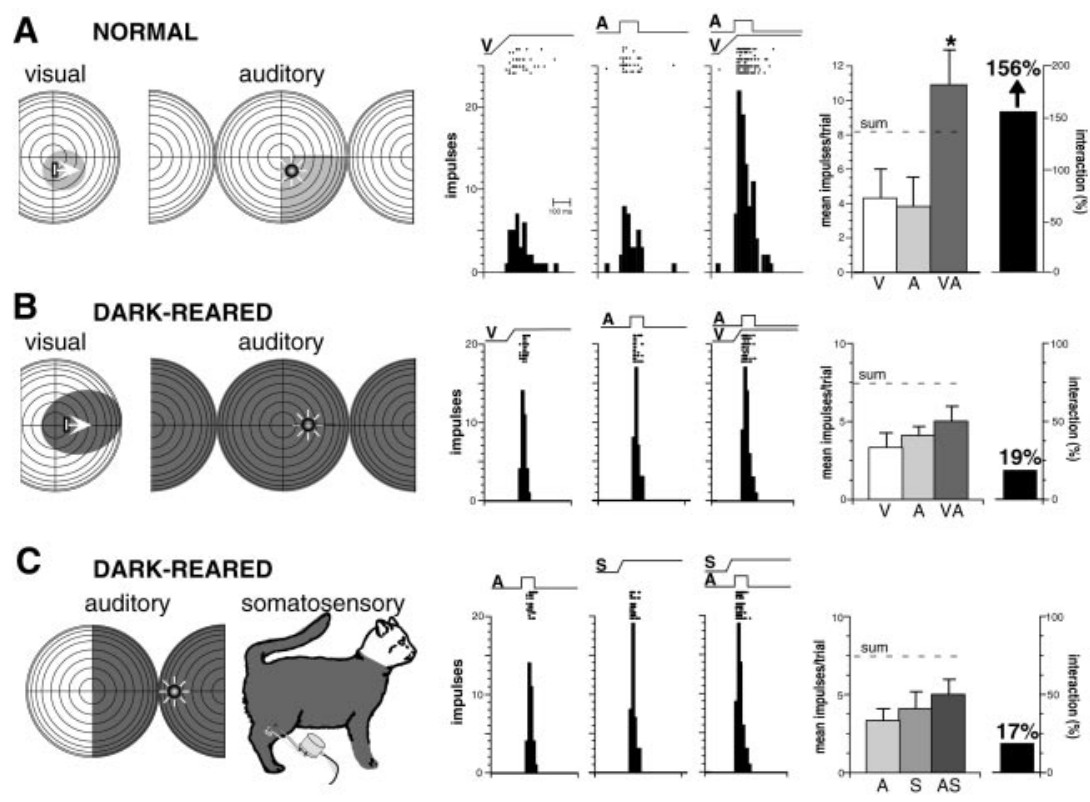

Figure 3. Multisensory integration, which is present in SC neurons in animals raised in a normal lighted environment $(A)$, is absent in animals raised in complete darkness $(B, C)$. On the left are shown the receptive fields (shading) of representative multisensory neurons, along with the locations of the stimuli (icons) used to evaluate multisensory responses (receptive field conventions are the same as in Fig. 2). In the middle, rasters and histograms show the responses of these neurons to both modality-specific and multisensory stimuli. Each dot in the raster represents an action potential, and each row of dots represents the response to a single stimulus repetition. In the histograms, each bin is $10 \mathrm{msec}$, and the ramps and square waves at the top denote the presentation of the stimuli. On the right, summary bar graphs illustrate the mean responses for each of the conditions and the magnitude of the multisensory interaction, calculated as $\left[\left(C M-S M_{\max }\right) /\left(S M_{\max }\right)\right] \times 100$, where $C M$ is the response to combined-modality stimulation, and $\mathrm{SM}_{\max }$ is the response to the more effective of the single modality stimuli. sum, Response predicted based on the addition of the two modality-specific responses. ${ }^{*} p<0.05$. A, Auditory; $S$, somatosensory; $V$, visual; VA, visual-auditory; AS, auditory-somatosensory. 


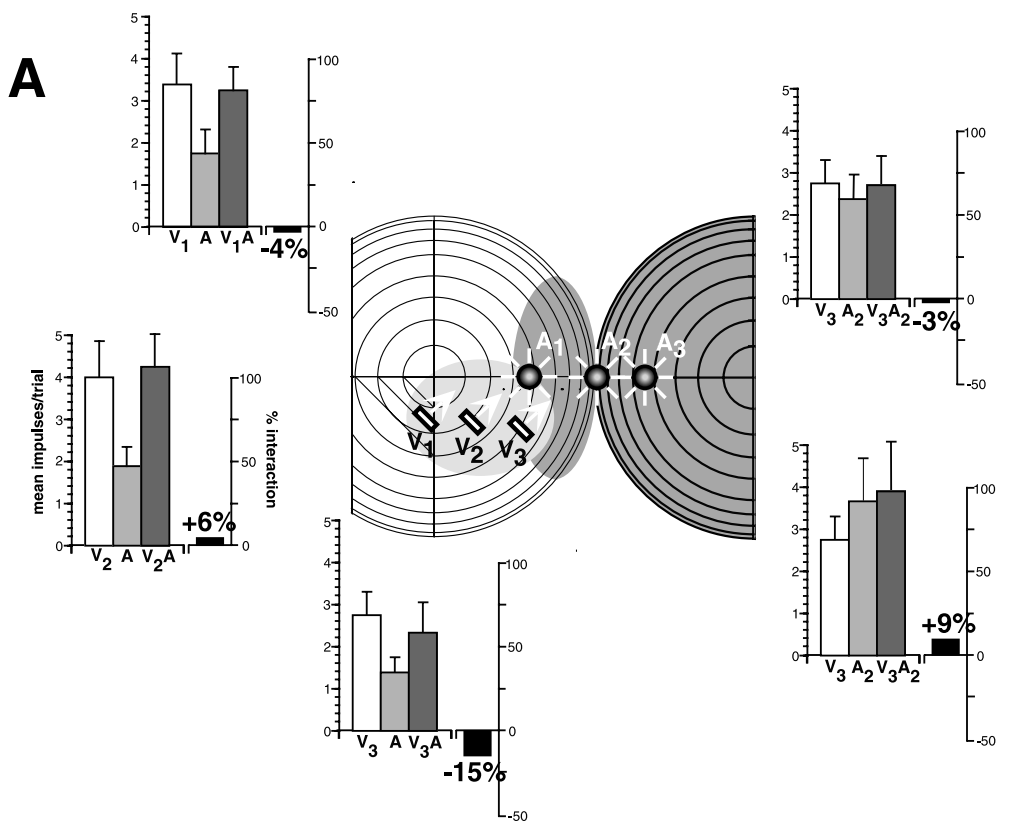

B
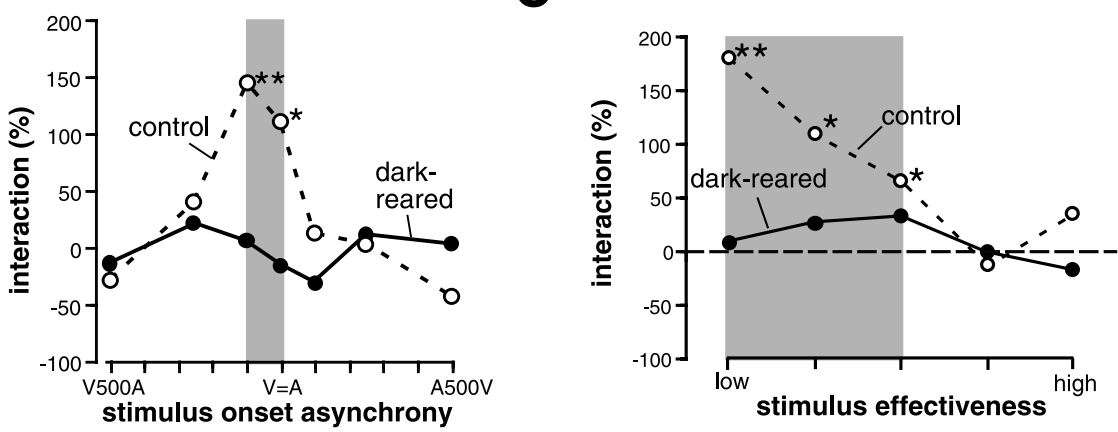

Figure 4. Regardless of their spatial or temporal relationship, or their level of effectiveness, multisensory stimuli failed to generate significant interactions in SC neurons of dark-reared animals. A, Manipulation of the spatial relationships of the visual and auditory stimuli in this example failed to produce a significant interaction. The central plot shows the receptive fields [visual (V), lighter shading; auditory (A), darker shading] and stimulus locations (i.e., $V_{1}, V_{2}, V_{3}, A_{1}, A_{2}, A_{3}$ ) used for these tests. Bar graphs surrounding the receptive field plots show the responses to the five different spatial combinations, as denoted below the bar graphs. $B$, Manipulation of the temporal relationships among the stimuli fails to produce multisensory interactions in darkreared animals. Plotted here is the multisensory interaction as a function of stimulus onset asynchrony for a visual-auditory neuron in a control (open circles, dashed lines) and dark-reared (filled circles, solid lines) animal. V500A represents the visual stimulus coming on 500 msec before the auditory stimulus; $V=$ A represents simultaneous onsets for the two stimuli, etc. Note the temporal window (gray shading) within which multisensory interactions are seen in the neuron from the control animal and the lack of such interactions in the neuron from the dark-reared animal. ${ }^{*} p<0.05 ;{ }^{* *} p<0.01$. C, Manipulation of stimulus effectiveness failed to produce significant interactions in dark-reared animals. Plotted here is the multisensory interaction as a function of the level of effectiveness of the two modality-specific stimuli. Note the presence of significant interactions in the SC neuron from the control animal at low levels of stimulus effectiveness (gray shading) and the lack of any interactions in dark-reared animals.

constraints within which such integration can occur. For example, given the failure of receptive fields to contract during development in these animals, the spatial extent over which an integrated response might occur could be different from that expected based on data from control animals. To examine this possibility, a series of tests was conducted in a subset of neurons $(n=11)$ that did not exhibit multisensory integration with stimuli that were placed in spatial arrangements that normally would yield response enhancements (i.e., spatially coincident pairings). Although each neuron was tested with an average of 4.7 different spatial stimulus configurations, in no case did these manipulations result in a significant multisensory interaction (Fig. 4A). A second possibility was that dark rearing altered the temporal profile of multisensory integration. To test this possibility, the tem- poral interval between the multisensory stimuli was systematically manipulated in 14 neurons that, like those above, did not show multisensory integration on initial tests when presented with spatially and temporally coincident stimuli. An average of 6.3 different stimulus onset asynchronies ranging from 500 to $0 \mathrm{msec}$ (i.e., synchronous onsets) were tested, but, once again, no multisensory integration was seen (Fig. 4B). A third possibility was that using weakly effective modality-specific test stimuli in an effort to maximize multisensory integration was the wrong strategy. Perhaps dark rearing negated the principle of inverse effectiveness, in which multisensory response enhancements are greatest when the individual component stimuli are weakest (Meredith and Stein, 1986). Indeed, there is considerable variability in this factor even among SC neurons in normal control animals (Perrault et al., 2003). Therefore, stimulus effectiveness was systematically manipulated in 17 neurons that did not show multisensory integration during initial tests. Although an average of 4.5 different levels of effectiveness were tested for each of these neurons, these tests again failed to yield a significant multisensory interaction (Fig. 4C).

Finally, to test the possibility that SC neurons in dark-reared animals had acquired the capacity for multisensory integration during development (Wallace and Stein, 1997) but had subsequently lost that capacity in the absence of visual experience (and that the small incidence of neurons with this capacity represented the residual of this population), an additional set of experiments was conducted in five animals at 2, 4, 6, 8, and 12 postnatal weeks of age. In these animals, 27 multisensory neurons were quantitatively examined using a series of tests in which the spatial and temporal relationships and the effectiveness of the cross-modal stimuli were manipulated. In each of these neurons, receptive fields were found to be very large and in no case was multisensory integration evident. These multisensory neurons (most of which were found at the later two ages) looked identical in their multisensory properties to neurons in animals raised until adulthood in the dark.

It is interesting to note that the few neurons $(n=7)$ in darkreared adults that did exhibit multisensory integration had receptive fields that were comparable in size with those seen in control adults and that these integrating neurons were encountered in animals that had experienced a minimum of eight recording sessions. Although deeply anesthetized during the entire recording session, all animals received some visual experience during the recording period and during a 1-2 hr recovery period that followed each experiment and preceded their return to their dark housing environment. Given that neither receptive field contrac- 
tion nor multisensory integration were evident in the 67 neurons studied before this period in dark-reared adults (including these same animals) and that no such properties were noted during any of the earlier developmental stages that were sampled in these and other animals, the data suggest that the visual experience gathered during the recovery period may have been sufficient to drive the maturation of the integrative capacity in these few neurons. Whether this is indeed the case, and whether this experience will prove to be as potent as that acquired early in life, remain to be determined.

\section{Discussion}

Together, these results suggest that, although sensory experience is not necessary for SC neurons to become responsive to sensory stimuli or to become responsive to stimuli from multiple sensory modalities, it is essential if they are to develop the capacity to synthesize information from these different modalities. This makes intuitive sense because the ability to integrate cross-modal information enables SC neurons to significantly modulate their activity and the behaviors that depend on it, based on the complexes of sensory stimuli that are encountered (Stein and Meredith, 1993). Experience could craft this process to ensure the enhanced salience of those stimulus complexes most important for the animal's survival. Thus, a unitary event that is signaled in two or more modalities could readily drive the development of receptive field register via a simple Hebbian-based mechanism. Once such receptive field register is established, the neuron is now primed for the capacity to integrate those inputs, a property that appears to be conferred via the maturation of extrinsic gating influences from the cerebral cortex (Wallace and Stein, 2000; Jiang et al., 2001). In the absence of such coactivation, such as what has been generated here by the absence of visual experience, the consolidation of receptive fields and the consequent appearance of strong receptive field register fails to take place, preventing the appearance of multisensory integration.

Several intriguing questions are brought to the fore by these observations. The first is whether or not the visual experience must be obtained during early development for normal multisensory integration to appear. Specifically, does the early absence of visual experience simply delay the appearance of multisensory integration until the requisite visual experiences are received? Such a possibility, which is in keeping with contemporary views of developmental plasticity within individual sensory systems (Katz and Shatz, 1996), awaits future study. A second question is the specificity of the lack of visual experience for the observed effects. Although there is substantial evidence for vision playing an instructive role in the development of multisensory representations (King et al., 1988; Knudsen and Brainard 1991), the consequences of a loss of auditory or somatosensory input for the development of multisensory processes remain essentially unknown. Germane to this issue is the observation in the current study that nonvisually responsive (i.e., auditory-somatosensory) neurons appear to be as susceptible to the impact of visual deprivation as their visually responsive counterparts, a finding suggestive of a possible preeminence of vision in the development of mature multisensory integration.

\section{References}

Bell AH, Corneil BD, Meredith MA, Munoz DP (2001) The influence of stimulus properties on multisensory processing in the awake primate superior colliculus. Can J Exp Psychol 55:123-132.

Benedetti F, Ferro I (1995) The effects of early postnatal modification of body shape on the somatosensory-visual organization in mouse superior colliculus. Eur J Neurosci 7:412-418.

Frens MA, Van Opstal AJ (1998) Visual-auditory interactions modulate saccade-related activity in monkey superior colliculus. Brain Res Bull 46:211-224.

Jiang W, Wallace MT, Jiang H, Vaughan JW, Stein BE (2001) Two cortical areas mediate multisensory integration in superior colliculus neurons. J Neurophysiol 85:506-522.

Kandel E, Jessell T, Sanes J (2000) Sensory experience and the fine-tuning of synaptic connections. In: Principles of neural science, Ed 4 (Kandel E, Schwartz J, Jessell T, eds), pp 1115-1130. New York: McGraw-Hill.

Katz LC, Shatz CJ (1996) Synaptic activity and the construction of cortical circuits. Science 274:1133-1138.

King AJ, Palmer AR (1985) Integration of visual and auditory information in bimodal neurones in the guinea-pig superior colliculus. Exp Brain Res 60:492-500.

King AJ, Hutchings ME, Moore DR, Blakemore C (1988) Developmental plasticity in the visual and auditory representations in the mammalian superior colliculus. Nature 332:73-76.

Knudsen EI, Brainard MS (1991) Visual instruction of the neural map of auditory space in the developing optic tectum. Science 253:85-87.

Meredith MA, Stein BE (1983) Interactions among converging sensory inputs in the superior colliculus. Science 221:389-391.

Meredith MA, Stein BE (1985) Descending efferents from the superior colliculus relay integrated multisensory information. Science 227:657-659.

Meredith MA, Stein BE (1986) Visual, auditory, and somatosensory convergence on cells in superior colliculus results in multisensory integration. J Neurophysiol 56:640-662.

Perrault Jr TJ, Vaughan JW, Stein BE, Wallace MT (2003) Neuron-specific response characteristics predict the magnitude of multisensory integration. J Neurophysiol 90:4022-4026.

Sanes D, Reh T, Harris W (2000) Development of the nervous system. San Diego: Academic.

Stein B, Meredith M, Huneycutt W, McDade L (1989) Behavioral indices of multisensory integration: orientation to visual cues is affected by auditory stimuli. J Cognit Neurosci 1:12-24.

Stein BE, Meredith MA (1993) The merging of the senses. Cambridge, MA: MIT.

Stein BE, Labos E, Kruger L (1973) Sequence of changes in properties of neurons of superior colliculus of the kitten during maturation. J Neurophysiol 36:667-679.

Stein BE, Huneycutt WS, Meredith MA (1988) Neurons and behavior: the same rules of multisensory integration apply. Brain Res 448:355-358.

Wallace MT, Stein BE (1997) Development of multisensory neurons and multisensory integration in cat superior colliculus. J Neurosci 17:2429-2444.

Wallace MT, Stein BE (2000) Onset of cross-modal synthesis in the neonatal superior colliculus is gated by the development of cortical influences. J Neurophysiol 83:3578-3582.

Wallace MT, Meredith MA, Stein BE (1993) Converging influences from visual, auditory, and somatosensory cortices onto output neurons of the superior colliculus. J Neurophysiol 69:1797-1809.

Wallace MT, Wilkinson LK, Stein BE (1996) Representation and integration of multiple sensory inputs in primate superior colliculus. J Neurophysiol 76:1246-1266.

Wallace MT, Meredith MA, Stein BE (1998) Multisensory integration in the superior colliculus of the alert cat. J Neurophysiol 80:1006-1010. 